\title{
Development of An Arabic Conversational Intelligent Tutoring System for Education of Children with ASD
}

\author{
Sumayh S. Aljameel, James D. O’Shea, Keeley A. Crockett, Annabel Latham, Mohammad Kaleem \\ Department of Computing, Math and Digital Technology \\ Manchester Metropolitan University \\ Sumayh.s.aljameel@stu.mmu.ac.uk,\{j.d.oshea,k.crockett, a.latham,m.kaleem\}@mmu.ac.uk
}

\begin{abstract}
This paper presents a novel Arabic Conversational Intelligent Tutoring System (CITS) that adapts the learning styles VAK for autistic children to enhance their learning. The proposed CITS architecture uses a combination of Arabic Pattern Matching and Arabic Short Text Similarity to extract the responses from the resources. The new Arabic CITS, known as LANA, is aimed at children with autism (10 to 16 years old) who have reached a basic competency with the mechanics of Arabic writing. This paper describes the architecture of LANA and its components. The experimental methodology is explained in order to conduct a pilot study in future.
\end{abstract}

Keywords - Autism Spectrum Disorder; Conversational Agent; Intelligent Tutoring System; String Similarity; The Arabic Language.

\section{INTRODUCTION}

Autism spectrum disorder (ASD) has four main diagnostic categories: Autistic Disorder, Asperger Syndrome, Pervasive Developmental Disorder and Childhood Disintegrative Disorder. This project has been specifically designed for highfunctioning individuals or Asperger's (i.e. those with higher verbal IQ). People with ASD have the ability to deal with technology and computers better than neurotypical people because of the structured environment that is offered by software. This environment is preferred by people with autism because it is more in-line with their routine and repetitive behaviour [1][2][3]. Traditional education using human tutors is a challenge for children with autism. Whereas virtual tutors can meet the individual children's needs. One advantage of using a virtual tutor is that some autistic children are uncomfortable looking at faces and human interaction. A virtual tutor's face can be made to look like a cartoon, which is less intrusive and more familiar to the child, and when the child becomes a custom with the virtual tutor, the complexity can be increased. There is some evidence to suggest that autistic children who were taught by virtual tutors gained more information compared to those who were taught by human tutors [4]. Another advantage is, the virtual tutor allows children to learn and practice their skills independently. Consequently, the human tutor can focus on the complex aspects and tasks while the software covers the general topics [5][6]. Children with autism tend to learn through one of three kinds of learning styles (VAK): through seeing (Visually), through hearing (Auditory), and through touching an object (Kinesthetically). Children with autism are more likely to rely on one style of learning, which is determined either by observation by a teacher or by using one of a number of behavioral questionnaires [7]. The most common of which is "VAK Learning Styles Self-Assessment Questionnaire" [8]. According to Papanikolaou et al.[32], Intelligent Tutoring Systems (ITS) are computerised learning systems that mimic human tutors to provide more personalised learning than previous content delivery systems. A Conversational Intelligent Tutoring Systems (CITS) is an extension of ITS. It integrates natural language interfaces rather than menus to allow users to learn topics through discussion as they would in the classroom. Many researchers developed CITS for different domains. AutoTutor [33] is one example of CITS; it is a computer tutor that is designed to assist students at university level to learn an introductory computer literacy course. The system teaches the fundamentals of hardware, operating systems, and the Internet. However, AutoTutor does not consider student learning styles during a tutoring session. This means that all students are provided with the same learning experience regardless their abilities. Oscar [34] is another example of CITS, which overcomes AutoTutor's limitations. Oscar leads a tutoring conversation and it dynamically predicts and adapts to a student's learning style. Oscar was developed for students at University level and teaches SQL. In the Arabic language, CITS is considered as a new area of research. Recently, Alobaidi et al. [15] developed an Arabic CITS (Abdullah) for modern Islamic education. Abdullah CITS is an online system that aims to teach children aged 10 to 12 years old the essential topics in Islam. The system allows conversation, discussion and interpretation with verses in classical Arabic language by engaging in dialogue using Modern Arabic language. To our knowledge, no academic research exists on Arabic conversational intelligent tutoring systems for Autistic children.

In this research, an Arabic CITS was developed called LANA. The intention behind the research and development of LANA was to support education for autistic children (10 to 16 years old) who have reached a basic competency with the mechanics of Arabic writing. The LANA CITS engages children with a science tutorial delivered in modern standard Arabic. The 
curriculum material that is delivered to students through LANA is mapped to the VAK model. The LANA architecture utilises pattern matching (PM) and Arabic short text similarity (STS) to extract the responses to user utterances from structured stored resources in particular domain (science). This project measures learning gain to evaluate the ability of a CITS to adapt to autistic children's learning styles. The current phase of the research focuses on two research questions:

1. Can an Arabic Conversational Intelligent Tutoring System (CITS) adapt to the learning styles of autistic children?

2. Can an Arabic Conversational Intelligent Tutoring System (CITS) for children with autism enhance their learning?

This paper is organised as follows: Section II describes the Conversational Agent and its approach. Section III contains the LANA architecture and its components. Section IV discusses the experimental methodology. Section $\mathrm{V}$ includes the conclusion and future works.

\section{CONVERSATIONAL AgENT}

\section{A. Background}

A Conversational Agent (CA) is defined as 'a computer program which interacts with a user through natural language dialogue and provides some form of service' [9]. According to Crockett et al. [10], A CA is defined as having 'the ability to reason and pursue a course of action based on its interactions with humans and other agents'. CAs have become a popular communication method and have been used in many applications in different domains such as e-commerce, medication, and education [11] [12]. Also, CAs are used effectively in many application such as student's debt management guidance [13], database interfacing [14], and as student's assistant by providing problem- solving advice as they learn [15]. There are two categories of CAs, 'Embodied CAs' (ECA) and linguistic CA's (LCA). Both categories are becoming increasingly popular in the field of CA. An embodied conversational agent (ECA) is defined as an agent which simulated face-to-face conversation with the user, by presenting the user with an avatar who talks to the user and uses the naturalistic modes of communication such as hand, head, facial expressions and body gestures [16]. A Linguistic conversational Agent (LCA) is defined as an agent who can converts with the user in written or spoken form [17].

\section{B. Conversation Agent Approaches}

There are three main approaches that are used to develop linguistic based CAs. These are Natural Language Processing (NLP); Pattern Matching (PM); and semantic sentence similarity measures (SSM), discussed briefly below.

Natural language processing (NLP) is defined as "the computational processing of textual materials in natural human languages" [18]. Researchers have been encourage to develop CAs based on NLP, focusing on extracting the utterance using grammar rules and list of attribute pairs from the conversation. This information is used to fill a template-based structure to generate a suitable response. Nevertheless, NLP based CAs emphasis on structured lexicon, parsing and various other language handling aspects without reasoning about the meaning of the utterance. According to Demir [19], an NLP-based CA should understand the user's utterance in order to produce human-like conversations, meaning the semantic content of expressed information should be recognized. There are certain other disadvantages of NLP-based CAs. First, the system might not recognise the misspelling of a word in the conversation and so the system may fail to understand the whole conversation. Second, NLP-based CAs may not comprehend dialogue due to natural mistakes in grammar that people often make in everyday conversation. Thirdly, natural language is very ambiguous due to the rich in form and structure, which means that the word might have more than one meaning (lexical ambiguity) or a sentence might have more than one structure (syntactic ambiguity) [20].

Text-based Pattern Matching (PM) is the process of measuring the degree of matching between a string or sequence of strings and a piece of text [21]. From a CA perspective, PM algorithms are used to handle user conversations by matching a CA's patterns against a user's utterance. A typical pattern is generally composed of wildcards, words and spaces. A wildcard is a symbol used to match a portion of the user's utterance [22]. InfoChat [23] as well as ALICE [24] are two examples of CAs incorporating PM algorithms. The typical procedure for such CAs is organizing a scripted domain into contexts (about a particular topic), each of which has a number of related rules. Each rule has structured patterns that represent a user's utterance. PM is considered one of the more successful methods for developing CAs [25]. PM is deemed to have various benefits. For example, it has reduced operating and computing costs, as well as requiring less time for processing, due to there being no need for intricate procedures prior to processing. Furthermore, PM is effective regardless of language and is straightforward to comprehend. As a result, web-based interactions and conversations that are happening live and involve many individuals can be analysed and aided through CAs based on PM method [26]. An additional advantage is that PM CAs are able to overcome many linguistic challenges, which the NLP approach faces, such as morphological changes occurring on words in the form of prefixes and suffixes. This is largely addressed through the utilisation of pattern wildcards that can amend mistakes in spelling and grammar, as well morphology amendments [27].

The main limitation of using PM based CAs is the needs for a large number of patterns to implement a coherent domain. This limitation causes many issues. For example, certain keywords may have multiple meanings as well as singular and plural configurations, thus a programmer will have to take in to account these various possibilities and innate linguistic depth in terms of morphology. If one were dealing with Arabic language, then its various morphological amendments, prefixes and suffixes can be compensated for utilising various methods, however the PM procedure can be particularly complex, especially in scripting. A further issue is that a user utterance may be written in multiple ways through adopting various alternative words and phrase structure.

Information, philosophy and linguistic theories have all been adopted as a means of assessing sentence similarity, which is 
the third approach for CAs [28]. Generally, similarity as a term is used to describe the similarity level between two objects. Semantics is "the study of the meaning of linguistic expressions" [29]. Information retrieval, question answering, information extraction machine translation, data mining machine translation, and conversational agents have all been aided by means of similarity-based research, while it also has wider application to AI and computer processing as a whole. Applying the sentence similarity technique in CA development is more effective than other techniques because it replaces the scripted patterns by a few natural language sentences in each rule. Consequently, the scripting effort and script size are reduced to a minimum. Thus, there is a reduction in the maintenance cost of such scripts [30] [31]. One limitation of using this approach is a lack of previous research in this area for Arabic language.

\section{LANA CITS ARCHITECTURE}

This section illustrates the design and development of LANA CITS architecture. LANA CITS architecture was designed based on three main components as shown in Figure 1, which are: component 1 (consisting of the system knowledge base which includes: the tutorial scripts, log file, user's profile, and general contexts), component 2 (The Arabic CA), and component 3 (the ITS). The following sections will describe each component in detail.

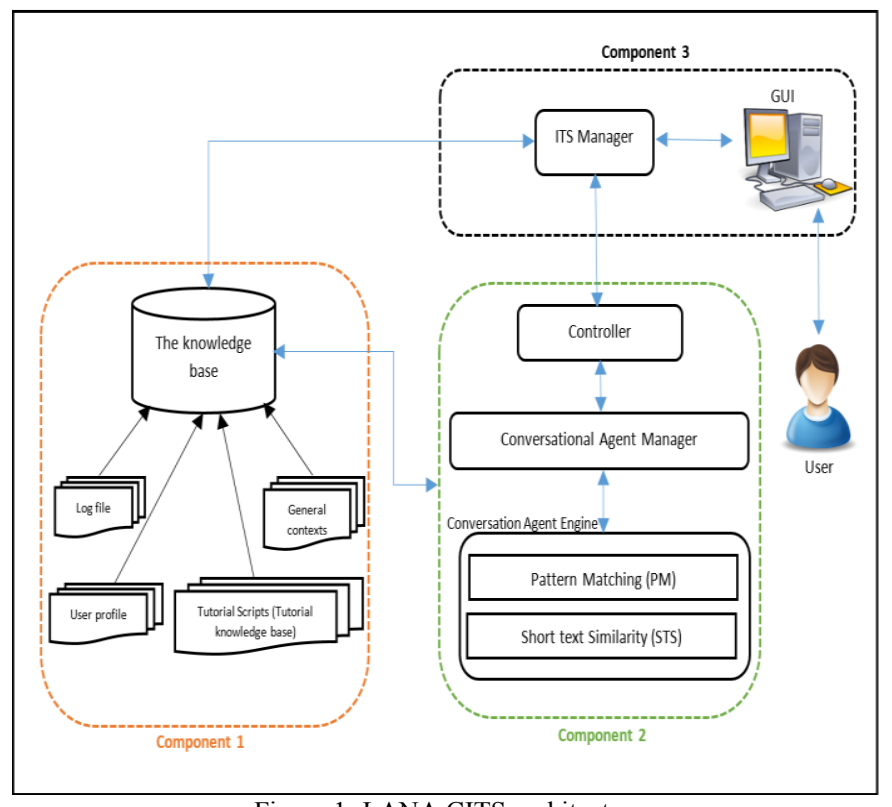

Figure 1: LANA CITS architecture

\section{A. Component 1: LANA CITS Knowledge Base}

The knowledge base consists of Tutorial Knowledge base, Arabic general contexts, user's profile and the log file. The Knowledge base design is draws on the knowledge engineering of the Arabic CA domain.

\section{The Domain}

The domain used to script the CA is concerned with teaching simple scientific topics such as the solar system to the targeted age group (10-16 years old). The scientific material is based on educational resources provided to schools by the Ministry of Education in Saudi Arabia. Scripts consist of a context structured according to the topics in the science book such as Earth, Moon, Solar System, the eclipse, etc.). LANA CITS is therefore designed to deliver the Science topics to Autistic learners in Arabic language based on standard teaching in schools in Saudi Arabia.

\section{Arabic CITS Knowledge Engineering}

Base on Jee et al.[35] the definition of, knowledge engineering is the process of extracting, representing, encoding, and testing of expert knowledge. Knowledge engineering was used in LANA CITS to script the Arabic CA domain, which involved a number of process beginning with extraction, design, and development of the knowledge base. In the knowledge extraction process, a short interview was conducted with three primary school teachers in Saudi Arabia, who have experience in teaching the science subject to the targeted age group. The aim of this interview was to design and deliver the tutorials in LANA CITS as closely as possible to how the teachers deliver the subject in the classes. The information garnered from this interview was transcribed and used to create the tutorials. In the knowledge design process, the information from the interviews was then collated and used with the science book to design the tutorials. The designed tutorial was evaluated by the teachers to get a feedback. Once the tutorials were approved by the teachers, the tutorials were implemented in LANA CITS. In the knowledge implementation process, the knowledge base was developed in a relational MySQL database in order to store and retrieve the resources. The knowledge base for the LANA CITS consists of 2 main layers: the domain, which is the science tutorials layer and, a general contexts layer, illustrated in Figure 2.

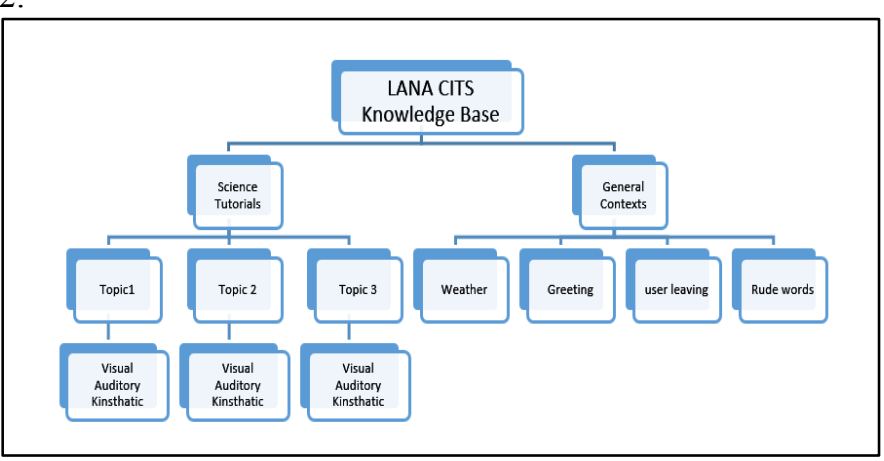

Figure 2: Knowledge base layers

Each layer represents a context, and each context has all the related sub contexts mapped to it. The domain layer holds all the tutorials sessions related to the science subject. The general contexts layer deals with general conversation not related to the domain, such as greeting and weather. The general context layer is included in to the knowledge base to make LANA CITS seem more intelligent by responding to the user's utterances, which are not related to the main domain. However, a few select sub-contexts have been implemented because it is not possible to cover all aspects of general contexts.

\section{Mapping the tutorial into VAK learning style model}

After the tutorial is designed and approved by the teachers then the tutorial questions are mapped to the VAK learning style 
model. Each tutorial question is associated with the VAK learning style material. Each question in the tutorial is adapted and mapped to the Visual learning style through pictures and videos, the Auditory learning style through the addition of more audio and, the Kinesthetic learning style through the introduction of models and instructions.

\section{Developing LANA CITS Scripting Language}

In order to script the domain, the domain is organised into a number of contexts and each context contains rules, each rule in the domain contains a number of patterns and a response that forms the CA output to the user. The contexts represent the tutorial topics and the rules represent the agent's questions for such topic, while the patterns represent the user's utterance, which belong to such rule. Table 1 shows an example of the scripting language.

\begin{tabular}{l}
\hline Context: 1 \\
Context Name: Topic 1 (Earth, Sun, and Moon) \\
\hline Rule Number: 1 \\
Rule Name: Object moves in the gravitationally curved path called \\
the orbit. The earth rotates around the sun and around its own axis. \\
How long the earth takes to rotate around its axis? \\
Pattern: It * a day \\
Pattern: It $*$ one day \\
Pattern: It $* 24$ hours \\
Pattern: * 24 hours \\
Response: Excellent, the earth takes one day (24 hours) to rotate \\
around its axis \\
Image: yes \\
Audio: yes \\
Instruction: yes \\
\hline
\end{tabular}

\section{B. Component 2: Designing and Implementing the Arabic CA}

The Arabic CA architecture illustrated in Figure 1 was implemented. The components were developed using JAVA. The knowledge base and script databases were developed using the MySQL. The functions of the individual components of the Arabic CA architecture are described in detail in the following sections.

\section{Controller}

The controller is responsible for directing and managing the entire conversation. The controller works with several other components, which are the ITS manager, GUI and CA manager to achieve the conversation goal. First, the controller communicates with the ITS manager to know the student's learning style. Second, it gets the user's utterance and provides an utterance checking process. The controller will check the utterance based on the following constraints:

- Cleansing the utterance: The controller uses the utterance filter to remove special characters (i.e. $\$, \&$, $*, !, ?$, “’, $£,(), \wedge)$ from the utterance.

- Check for rude words: the system will warn the user three times before terminating the session.

After the utterance is parsed the controller then works together with the conversation agent manager to ensure that the conversation is within the tutorial scenario, or whether the user switched the context. After that the controller delivers the response back to the user with the supporting material such as picture, audio, or instructions according to the user's learning style.

\section{Conversational Agent Manager}

The role of the Conversation Manager is to control the conversation to make sure that the goal is achieved, which is completing the tutorial. The conversation manager checks whether the user stays on the tutorial topic, or the user switch the context. Goal-oriented CAs should manage utterances in an intelligent way [36]. For example, when the CA manager receives an utterance that is not related to the tutorial topic, the CA manager checks the user utterance with the general contexts layer to see if the utterance matches other general contexts within the database. If the utterance matches one of these contexts, the CA manager generates the response to the user, and then the user is brought back to the point where the conversation is interrupted and then directed towards the goal.

\section{Conversational Agent Engine}

The LANA CA engine has two main components that were specifically developed to deal with the features unique to the Arabic language in terms of its grammatical and morphological nature. These components are: Pattern matching approach (Wildcard PM) and String similarity algorithm (Cosine algorithm). The combination of these components within LANA CA engine will help to calculate the similarity of the user utterance with scripted patterns using string similarity metrics. Consequently, reducing the need to cover all possible utterances when scripting the domain.

The first component of the Arabic CA engine, which is the primary phase of the engine, is pattern matching. It is based on similar Method to the Info Chat [23] of PM, where the user utterance is matched to stored patterns. These patterns contain wild card characters to represent any number of words of characters. For example, the wild card symbol (*) matches any number of words, where the wild card symbol (?) matches a single character. The second component of the Arabic CA engine is using the short text similarity algorithm, which is Cosine similarity [37]. It determines the similarity between two pieces of text by representing each piece of text in the form of word vector. A word vector is a vector of length $\mathrm{N}$ where $\mathrm{N}$ is the number of different tokens in the text. The similarity is computed as the angle between the word-vectors for the two sentences in vector space. For two texts $t 1$ and $t 2$ the cosine similarity is illustrated in the following equation:

$$
\operatorname{SIM}\left(t_{1}, t_{2}\right)=\frac{\sum_{i=1}^{n} t_{1_{i}} t_{2_{i}}}{\sqrt{\sum t_{1_{i}}{ }^{2}} \times \sqrt{\sum t_{2_{i}}{ }^{2}}}
$$

The cosine similarity result is non negative and bounded between $[0,1]$ where 1 indicates that the two text are identical.

\section{LANA CA Engine Workflow}

First the system will use PM (with wildcards) to match the user utterance with the pattern saved in the database, if a match is 
not found, the next step is to use STS Cosine similarity. To illustrate the cosine similarity algorithm, assume the user utterance was (S1), while the pattern stored in the LANA database was (S2). As shown in Table 2:

\begin{tabular}{|c|c|}
\hline 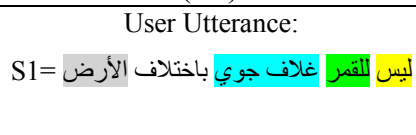 & $\begin{array}{c}\text { S1: The moon does not have } \\
\text { atmosphere whereas the earth } \\
\text { has. }\end{array}$ \\
\hline $\begin{array}{c}\text { Stored pattern in LANA CITS: } \\
\text { S2= يختلف القمرعن الارض بأن ليس له } 2 \text { يلاف جوي }\end{array}$ & $\begin{array}{l}\text { S2: The moon is different from } \\
\text { the earth that it does not have } \\
\text { an atmosphere. }\end{array}$ \\
\hline
\end{tabular}

Table 2: Cosine Similarity Example

The result obtained from Cosine similarity when applied to S1 and S2 is equal (0.95). The utterance is not recognised by the PM approach, whereas when the system applied the Cosine similarity algorithm, the utterance is recognised.

\section{Component 3: LANA Intelligent Tutoring System (ITS)}

This section describes the third component in LANA CITS architecture, which contains two main components, The Graphics User Interface, and The ITS manager.

\section{Graphics User Interface}

The GUI is the point where LANA CITS and the user interact with each other. LANA CITS has a character called LANA, which appears throughout the system interfaces in order to make it more engaging and natural for the users. Massaro and Bosseler [38] developed a computerized tutor called (Baladi) to teach vocabulary and functional language use to children with Autism with and without a cartoon face. They evaluated whether the face would increase the rate of learning for receptive measures. The results showed that the children's performance with the face condition was better than in the no face condition. Based on this, LANA's character was designed and evaluated by primary school teachers in order to make the tutorial more engaging. Figure 3 shows screenshot of the LANA GUI.

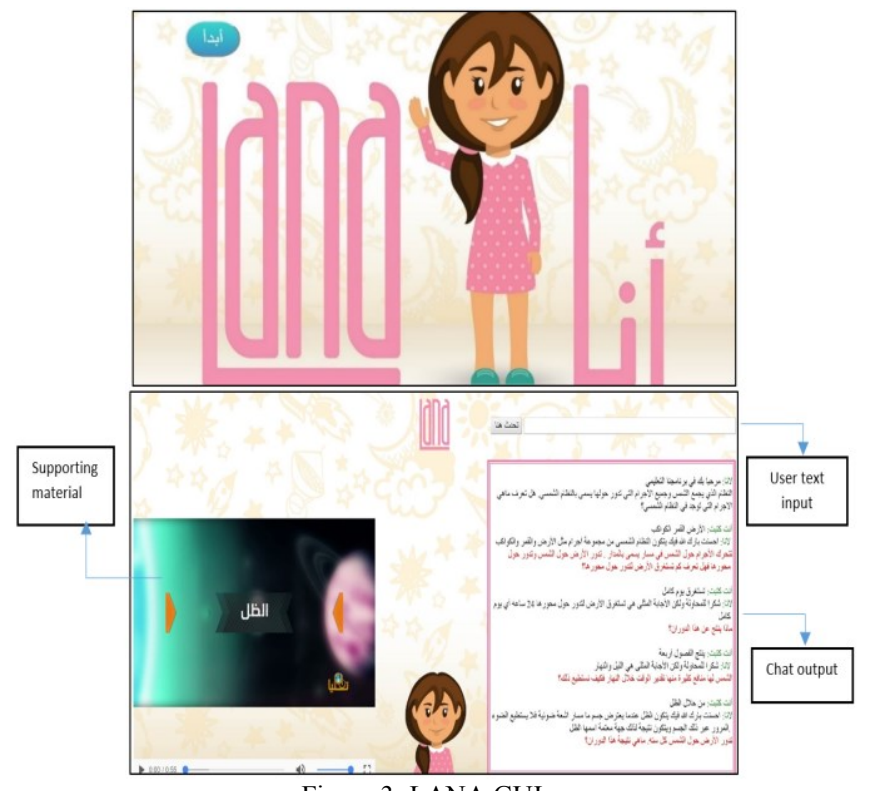

Figure 3: LANA GUI

\section{ITS Manager}

The ITS manager is responsible for personalising the tutorial according to the user's learning style, which is determined at the beginning of the tutorial through a questionnaire. The ITS manager can interact with: the conversation agent components through the controller, the Knowledge base, and the Graphics user interface. In order to personalise the tutorial session according to the student's learning style, a questionnaire will be applied first for each child. The questionnaire was developed on the basis of a widely disseminated version [39] to be completed by pupil with parents or teacher help. This questionnaire focused on Smith's visual, auditory, and kinaesthetic styles (VAK). There were three questions for each style and the pupils had to respond 'yes' or 'no' to each question. There are a total of nine questions in the questionnaire and the student's learning style result will be based on the highest score in one area.

\section{EXPERIMENTAL METHODOLOGY}

In order to evaluate the implemented LANA CITS framework and its architecture, a pilot study will be conducted with Nonautistic children (age 10-16 years old). This pilot study aims to evaluate the effectiveness and robustness of LANA CITS in order to address any weakness and then to conduct the second study with Autistic children. To formulate evaluation metrics, the Goal Question Metric (GQM) methodology is used [40]. The (GQM) model is used to identify which metrics are required to gauge the effectiveness and robustness of LANA. A total of 24 participants will be involved with age group (10-16) years old and their first language is Arabic. The evaluation will be done through two experiments: The first experiment is to test two questions, which are:

- Question1: Can the learning Style VAK help the student to learn better?

- Question2: Can LANA CITS adapt the student's learning style?

The data will be gathered from the user feedback questionnaire and the pre- and post-test scores with and without using the VAK learning Style. The second experiment will be conducted to test two questions, which are:

- Question 1: Are the participants satisfied with the usability of LANA CITS?

- Question 2: is LANA CA robust?

The data will be gathered from the LANA log file and the user feedback questionnaire.

\section{CONCLUSION AND FUTURE WORKS}

This paper outlined a novel Arabic CITS called LANA, which used VAK learning style model to enhance the learning for children with Autism. LANA CITS has the main features:

- $\quad$ LANA CITS will be able to adapt the VAK learning style within the tutorial.

- LANA CITS will be able to converse in Arabic language to teach children age (10-16) the science subject. 
- LANA engine will use PM with STS similarity algorithm to recognise the user utterance.

In future, a first pilot study will be conducted to collect preliminary data, using the experimental methods that were mentioned in the previous section. The pilot study aims to test the proposed architecture, and identify any details that need to be addressed before conducting the second pilot study with Autistic children on the target age group (10-16) years old whose first language is Arabic, who have high functioning autism (HFA) and have no problem with language and intellectual disabilities. The combination of the two testing stages will ensure the robustness and tutoring success of the final CITS.

\section{REFERENCES}

[1] C. Putnam and L. Chong, "Software and technologies designed for people withautism: what do users want?," presented at the 10th International ACM SIGACCESS conference oncomputers and accessibility, 2008.

[2] D. Murray, Autism and information technology: Therapy with computers. (In Autism and Learning: A Guide to Good Practice). Brookes, 1997.

[3] S. Parsons and S. Cobb, "State-of-the-art of virtual reality technologies for children on the autism spectrum," European Journal of Special Needs Education, vol. 26, no. 3, pp. 355-366, 2011.

[4] O. Grynszpan, J.-C. Martin, and J. Nadel, "Multimedia interfaces for users with high functioning autism: An empirical investigation," International Journal of Human - Computer Studies, vol. 66, no. 8, pp. 628-639, 2008

[5] D. Massaro, "Symbiotic Value of an Embodied Agent in Language Learning," presented at the Proceedings of the 37th Hawaii International Conference on System Sciences, 2004.

[6] E. 1. Limoges, L. Mottron, and C. B. Bolduc, Claude Godbout, Roger, "Atypical sleep architecture and the autism phenotype," Brain, vol. 128, 2005.

[7] F. Baker. (2015). Learning styles in children. Available: http://www.kidspot.com.au/school/primary/learning-andbehaviour/learning-styles-in-children

[8] V. Chislett. (2015). VAK Learning Styles Self-Assessment Questionnaire. Available: Http://www.businessballs.com/vaklearningstylestest.htm

[9] J. O'Shea, Z. Bandar, and K. Crockett, "Systems Engineering and Conversational Agents," 2011, vol. 10, pp. 201-232.

[10]K. Crockett, O. S. James, and Z. Bandar, "Goal orientated conversational agents: applications to benefit society," in Agent and Multi-Agent Systems: Technologies and Applications: Springer, 2011, pp. 16-25.

[11]A. Campbell, "TECHNOLOGY ASSISTED SEXUAL HEALTH AGENT (TASHA): A CONVERSATIONAL AGENT FOR PATIENT DECISION MAKING ON THE MANAGEMENT OF CERVICAL DYSPLASIA/CANCER," in Making Difficult Clinical and Policy Decisions: The Example of Ageing and End of Life Care in Asia-Pacific, 2016: Smdm.

[12]S. Tegos, S. Demetriadis, and A. Karakostas, "Promoting academically productive talk with conversational agent interventions in collaborative learning settings," Computers \& Education, vol. 87, pp. 309-325, 2015.

[13]K. O'Shea, K. Crockett, and Z. Bandar, "Application of a semantic-based conversational agent to student debt management," in Fuzzy Systems (FUZZ), 2010 IEEE International Conference on, 2010, pp. 1-7: IEEE.

[14] M. Owda, Z. Bandar, and K. Crockett, "Information extraction for SQL query generation in the conversation-based interfaces to relational databases (C-BIRD)," in Agent and Multi-Agent Systems: Technologies and Applications: Springer, 2011, pp. 44-53.

[15]O. G. Alobaidi, K. A. Crockett, J. D. O'Shea, and T. M. Jarad, "Abdullah: An Intelligent Arabic Conversational Tutoring System for Modern Islamic Education," in Proceedings of the World Congress on Engineering, 2013, vol. 2.

[16]J. Cassell, Embodied conversational agents. MIT press, 2000.

[17]L. Yin, T. Bickmore, and D. E. Cortés, "The impact of linguistic and cultural congruity on persuasion by conversational agents," in International Conference on Intelligent Virtual Agents, 2010, pp. 343349: Springer.
[18]D. Crystal, "Front Matter," A Dictionary of Linguistics and Phonetics, Sixth Edition, pp. i-xxv, 2008.

[19]Ş. Demir, "Improved treatment of word meaning in a Turkish conversational agent," Citeseer, 2003.

[20] M. Fernezelyi, Z. S. Maszák, and R. Langh, "Smalltalk: interactive installation," in Proceedings of the 14th ACM international conference on Multimedia, 2006, pp. 1025-1026: ACM.

[21] M. Hijjawi, Z. Bandar, and K. Crockett, "The Enhanced Arabchat: An Arabic Conversational Agent," International Journal of Advanced Computer Science and Applications, 2016.

[22] M. Kaleem, J. O'Shea, and K. Crockett, "Development of UMAIR the Urdu Conversational Agent for Customer Service," 2014.

[23]D. Michie and C. Sammut, "Infochat scripter's manual," Convagent Ltd., Manchester, 2001.

[24]R. S. Wallace, "ALICE: Artificial Intelligence Foundation Inc," ed, 2008.

[25]T. Bickmore and T. Giorgino, "Health dialog systems for patients and consumers," Journal of biomedical informatics, vol. 39, no. 5, pp. 556$571,2006$.

[26] J. F. Allen, D. K. Byron, M. Dzikovska, G. Ferguson, L. Galescu, and A. Stent, "Toward conversational human-computer interaction," $A I$ magazine, vol. 22, no. 4, p. 27, 2001.

[27] M. Maragoudakis, B. Kladis, A. Tsopanoglou, K. Sgarbas, and N. Fakotakis, "Natural language in dialogue systems. A case study on a medical application," in Proceedings of the Pan-Hellenic Conference, with International Participation, in Human-Computer Interaction, PCHCI, Patras Greece, 2001, pp. 197-201.

[28] V. Hatzivassiloglou, J. L. Klavans, and E. Eskin, "Detecting text similarity over short passages: Exploring linguistic feature combinations via machine learning," in Proceedings of the 1999 joint sigdat conference on empirical methods in natural language processing and very large corpora, 1999, pp. 203-212: Citeseer.

[29] N. Y. Habash, "Introduction to Arabic natural language processing," Synthesis Lectures on Human Language Technologies, vol. 3, no. 1, pp. 1-187, 2010.

[30] Y. Li, Z. Bandar, D. McLean, and J. O'Shea, "A Method for Measuring Sentence Similarity and ilts Application to Conversational Agents," in FLAIRS Conference, 2004, pp. 820-825.

[31] J. O'Shea, Z. Bandar, K. Crockett, and D. McLean, "A comparative study of two short text semantic similarity measures," in KES International Symposium on Agent and Multi-Agent Systems: Technologies and Applications, 2008, pp. 172-181: Springer.

[32] K. A. Papanikolaou, M. Grigoriadou, H. Kornilakis, and G. D. Magoulas, "Personalizing the Interaction in a Web-based Educational Hypermedia System: the case of INSPIRE," User modeling and user-adapted interaction, vol. 13, no. 3, pp. 213-267, 2003.

[33] A. C. Graesser, P. Chipman, B. C. Haynes, and A. Olney, "AutoTutor: An intelligent tutoring system with mixed-initiative dialogue," IEEE Transactions on Education, vol. 48, no. 4, pp. 612-618, 2005.

[34] A. Latham, K. A. Crockett, and Z. Bandar, "A Conversational Expert System Supporting Bullying and Harassment Policies," in ICAART (1), 2010, pp. 163-168: Citeseer.

[35] B. D. Jee et al., "Drawing on experience: how domain knowledge is reflected in sketches of scientific structures and processes," Research in Science Education, vol. 44, no. 6, pp. 859-883, 2014.

[36] A. M. Latham, "Personalising learning with dynamic prediction and adaptation to learning styles in a conversational intelligent tutoring system," Manchester Metropolitan University, 2011.

[37] G. Qian, S. Sural, Y. Gu, and S. Pramanik, "Similarity between Euclidean and cosine angle distance for nearest neighbor queries," in Proceedings of the 2004 ACM symposium on Applied computing, 2004, pp. 1232-1237: ACM.

[38]D. W. Massaro and A. Bosseler, "Read my lips: The importance of the face in a computer-animated tutor for vocabulary learning by children with autism," Autism, vol. 10, no. 5, pp. 495-510, 2006.

[39] A. Smith, Accelerated learning in practice. A\&C Black, 1998.

[40]N. E. Fenton and S. L. Pfleeger, "Software Metrics: A Rigorous and Practical Approach: Brooks," ed: Cole, 1998. 